\title{
Theme: Gastroenterology
}

\section{Appendicitis in children with ventriculo-peritoneal shunt (Arch Dis Child. 2019;104:607-9)}

Whether to remove or leave the ventriculo-peritoneal (VP) shunt in situ, in a child with acute appendicitis, is often a perplexing issue. In this review of 6 reports, the authors tried to find the best strategy in cases with perforated and non-perforated acute appendicitis in children with VP shunt. Out of 12 children with perforated appendicitis, in whom the shunt was left in situ, complications were encountered in 6 cases (3 required externalization, two required conversion to ventriculo-atrial (VA) shunt and one had shunt discontinued). In group of 16 children with non-perforated appendicitis, complications necessitated externalization in one, conversion to VA shunt in one, and only antibiotic therapy in one subject with fluid in pouch of Douglas. These data suggest that the risk of ascending shunt infection is low in non-perforated appendicitis while in cases of perforated appendicitis, the risk of shunt infection and other complications appears higher. In the case of perforated appendicitis, the risk of developing a shunt infection secondary to peritonitis must also be balanced against the risk of revising the shunt. The limited data in these studies suggest shunt removal may not be mandatory in patients with appendicitis, particularly if the appendix is not perforated, but needs careful consideration based on the clinical and microbiological findings.

Fecal microbiota transplant in recurrent Clostridium difficile infections ( $\mathrm{J}$ Pediatr Gastroenterol Nutr. 2019;68:343-7)

Fecal microbiota transplant (FMT) is known to be curative in up to $91 \%$ cases of recurrent Clostridium difficile infection in noninflammatory bowel disease patients. However, the same beneficial effect has yet not been demonstrated in children with inflammatory bowel disease (IBD). This retrospective review involved 8 children with IBD (all on vancomycin) in association with recurrent $C$. difficile disease, who underwent fecal microbiota transplant by colonoscopy. Two children had recurrence by 2 months while another 3 had recurrence between 2 and 6 months. The median time of recurrence was 101 days. With a cure rate of $75 \%$ at 60 days, FMT administered for the treatment of recurrent $C$. difficile disease was thus proposed to be an effective short-term treatment option in pediatric IBD. However, there appears to be a significant rate of late recurrence of $C$ difficile infection after 60 days in these patients, signifying need for developing more effective and optimal management strategy for these children.

Does Dientamoeba fragilis cause abdominal pain or diarrhea in children? (Arch Dis Child. 2019 Feb 23. doi:10.1136/archdischild-2018-316383 [Epub ahead of print])

Dientamoeba fragilis, a flagellate protozoan parasite, has often been linked to several gastrointestinal symptoms, including diarrhea and abdominal pain. However, conclusive evidence for its role in causation of gastrointestinal pathologies is yet elusive. This study aimed at comparing the prevalence of $D$. fragilis (detected by real-time PCR) in stools of 200 children with chronic abdominal pain and diarrhea with that in 122 reference children from healthy community. The concentration of fecal calprotectin (a marker of intestinal inflammation) was compared in the two groups to demonstrate if presence of $D$. fragilis was causally associated with intestinal inflammation. D. fragilis was detected in $45 \%$ of patients and in $71 \%$ of healthy children. Median (IQR) concentrations of calprotectin in patients and healthy children with a positive PCR result were not different from those with a negative PCR result (40 (40-55) $\mu \mathrm{g} / \mathrm{g} v s .40$ (40-75) $\mu \mathrm{g} / \mathrm{g}$, respectively). Authors concluded that $D$. fragilis colonization is very much prevalent in healthy children, and is not associated with an increase in faecal calprotectin concentration, supporting the inference that it is not a pathogenic parasite. Routine testing for $D$. fragilis in children with chronic abdominal pain should therefore not be encouraged at all.

丹

Anal intrasphincteric botulinum toxin injection in Hirschsprung disease ( $\mathrm{J}$ Pediatr Gastroenterol Nutr 2019;68:527-32)

In Hirschsprung disease (HD), despite successful surgical treatment, $50 \%$ of children continue to experience chronic functional obstructive symptoms, attributable to a nonrelaxing anal sphincter complex. This study aimed at determining the efficacy of intrasphincteric botulinum toxin (BT) injection in relieving these distressing symptoms. Fifteen patients (median age $4 \mathrm{y}$ ) with $\mathrm{HD}$ and postoperative functional intestinal obstructive symptoms received neurostimulation-guided intrasphincteric BT injections, for specific delivery of BT to muscular fibers of the nonrelaxing anal sphincter complex. The Bristol stool form scale was used to assess stool consistency, and the Jorge-Wexner (JW) score to assess fecal continence. In the short-term, a significant improvement in stool consistency was noted in 12 of 14 patients, and JW score significantly decreased for 14 of 15 patients. In the medium-term, JW score significantly decreased for all patients, with an improvement of $50 \%$ or more for 10 patients. In the long-term, $83.3 \%$ of patients had normal stool consistency and JW score was $<3$ for all. Recurrent enterocolitis decreased from $86.7 \%$ to $8.3 \%$. A complete resolution of all symptoms without further medication was observed in $66.7 \%$ of patients in the long-term. Authors concluded that the intrasphincteric BT injection is a safe, effective, and durable option for the management of postoperative functional intestinal obstructive symptoms in Hirschsprung disease.

Vyom AgGarwaL drvyomaggarwal@gmail.com 